$E U R O P E A N$
PSYOCH

Volumen 6

Número 6

SEPTIEMBRE

EDICIÓN ESPAÑOLA

REVISTA DE LA ASOCIACIÓN EUROPEA DE PSIQUIATRÍA

352

ARTÍCULOS ORIGINALES

1999

Comportamiento automutilador de pacientes psiquiátricos hospitalizados

H. L. I. Nijman, et al

362

Detección de la ansiedad y la depresión en pacientes mayores hospitalizados utilizando un inventario breve

P. Huber, et al

370 Uso de ansiolíticos e hipnóticos en 376 pacientes psiquiátricos hospitalizados

P. Hardy, et al

381 Una comparación doble ciego de sertralina y. fluoxetina en el tratamiento de episodio depresivo mayor en pacientes ambulatorios D. Sechter, et al

PERSPECTIVAS

392

Estadios de adaptación a un diagnóstico médico de una condición somática grave J. E. Mękarski, et al

COMUNICACIÓN BREVE

400 Anomalías en el movimiento ocular sacádico en la demencia

H. J. Schewe, et al 


\section{En el camino}

de la reintegración

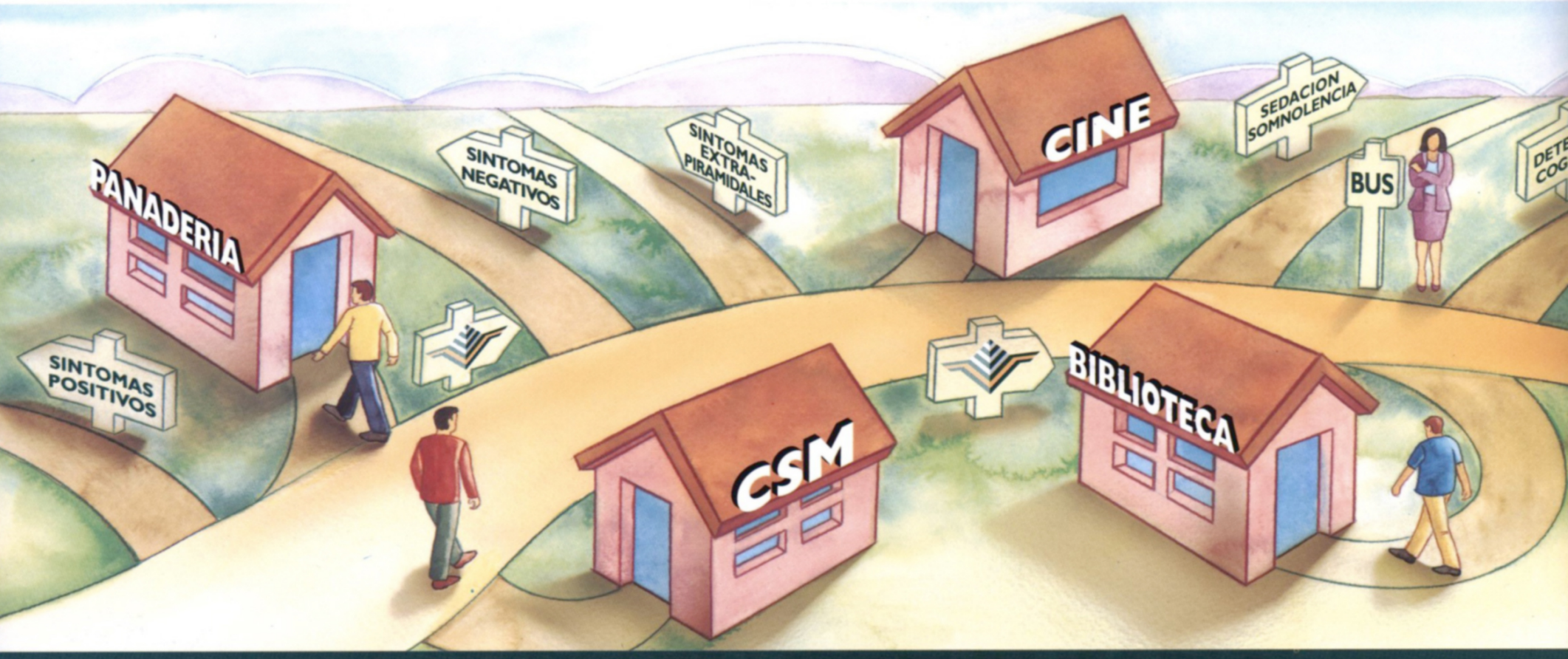

$\nabla /$ Eficaz en el control de sintomas positivos, negativos $y$ afectivos

$\mathbb{Y}$ Baja incidencia de efectos extrapiramidales

Baja incidencia de sedación

Mínimo aumento de peso

Ausencia de efectos anticolinérgicos 


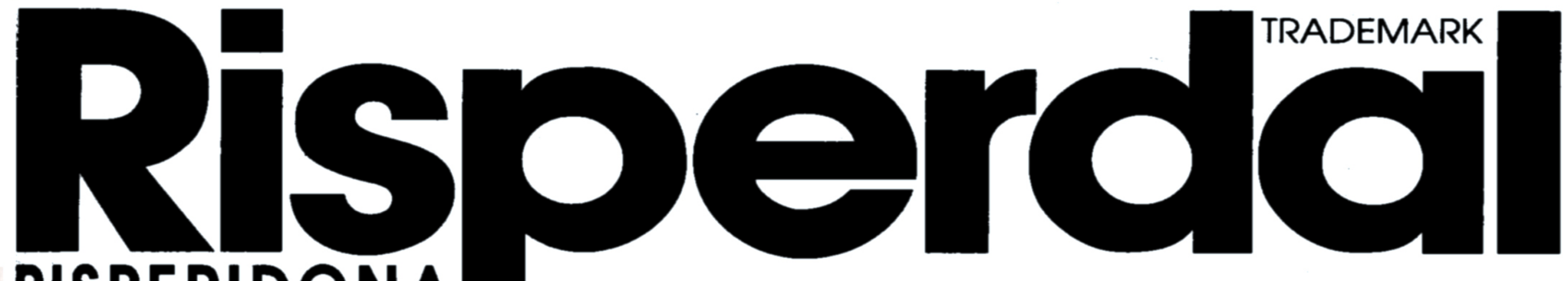
RISPERIDONA

\section{El antipsicótico de primera línea}
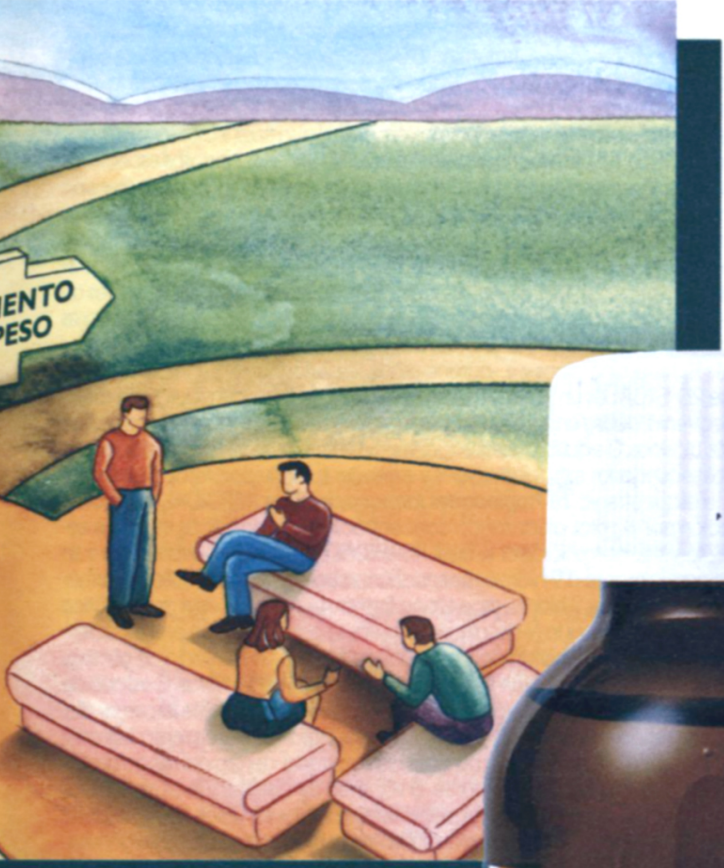

$100 \mathrm{ml}$ solución oral $659813 \mathrm{ct}$

Risperdal solución oral

risperidona, $1 \mathrm{mg}$ por $\mathrm{ml}$

Via oral

no congelar / guárdese entre $15^{\circ}$ y $30^{\circ} \mathrm{C}$

manténgase fuera del alcance de los ninos

Cida ml contiene:

exciplentidona (D.C.II)...1 mg

Registrado en la D.G.F.P.S con el $n^{\circ}: 62.096$

7. JANSSEN-CILAG

|IIIIIIII

El único antipsicótico atípico en Solución Oral

Rispercal $3 \mathrm{~m}$

60 comprimidos

7 JANSSEN-CILAG

$692145 \bigcirc$

con receta médica

9 


\section{泫 Risperdal}

1. Nombre del medicamento: RISPERDAL (Risperidona) 2. Composición cualitativa y cuanititativa: RISPERDAL $1 \mathrm{mg}$. comprimidos: Risperidona $1 \mathrm{mg} /$ comprimido RISPERDAL $3 \mathrm{mg}$. comprimidos: Risperidona $3 \mathrm{mg} / \mathrm{com}$ primido RISPERDAL solución oral: Risperidona $1 \mathrm{mg} / \mathrm{ml}$ 3. Forma formacéutica: Comprimidos recubiertos orales: 1 mg de risperidona como comprimido oblongo, ranurado, bianco. 3 mg de risperidono como comprimido oblongo, ranurado, amarilio. Solución oral: $1 \mathrm{mg}$ de risperidona por mililitro. 4. Datos clínicos: a) indicaciones ferapéuticas: RISPERDAL está indicado en el tratamiento de la psicosis esquizofrénicas agudas y crónicas, así como en otras condiciones psicóticas en las cuales los síntomos positivos (tales como alucinaciones, delirios, trastornos del pensamiento, hostilidad, recelo) y/o sintomas negativos (tales como afectividod embotada, aislamiento social y emocional, pobreza de lenguaje) sean notables. RISPERDAL también alivia los sintomas afectivos (tales como depresión, sentimientos de culpabilidad, ansiedad) asociados a la esquizofrenia. b) Posología y forma de administración: RISPERDAL está disponible en comprimidos y solución oral. Cuando RISPERDAL va a reemplazar a otros antipsicóticos, se recomienda si es apropiado desde el punto de vista médico, interrumpir de forma gradual el tratamiento previo, al mismo tiempo que se inicia la terapic con RISPERDAL. También y si es adecuado desde el punto de vista médico, cuando se trate de interrumpir un tratamiento con antipsicótiocos depot, se puede iniciar la terapia con RISPERDAL, reemplazando la siguiente inyección programada. Se debe evaluar periódicamente la necesidad de continuar con la medicación antiparkinsoniana que ya existía. Adultos RISPERDAL se puede administrar una vez al día o dos veces al dia. Se debe ajustar la dosis hasta $6 \mathrm{mg}$, de forma gradual a lo largo de tres dias. Iodos ios pacientes tanto los que presentan esquizofrenia aguda como crónica, deben comenzar con $2 \mathrm{mg} /$ dia de RISPERDAL. En el segundo día se incrementará la dosis hasta $4 \mathrm{mg}$, y en el tercer día hasta $6 \mathrm{mg}$. Después de esto la dosis se puede mantener sin cambios o bien individualizarse, si fuera necesario. La dosis óptima habitual está entre 4 y $8 \mathrm{mg} /$ dia. Sin embargo, algunos pacientes pueden beneficiarse de dosis inferiores. Un ajuste de dosis mós lento puede ser clínicamente adecuado. Las dosis por encima de $10 \mathrm{mg} / \mathrm{dia}$, no han demostrado ser más eficaces que las dosis más bajas y pueden ser causa de síntomas extrapiramidales. Dado que no se ha evaluado la seguridad para dosis mayores de $16 \mathrm{mg} /$ día, no se deben utilizar dosis por encima de este nivel.Si adicionalmente se requiere un efecto sedante puede administrar RISPERDAL con una benzodiacepina. Ancianos Se recomienda una dosis inicial de $0,5 \mathrm{mg}$ dos veces al dia. Esta dosis puede individualizarse mediante incrementos de $0.5 \mathrm{mg}$, 2 veces al dia, hasta $1-2 \mathrm{mg}$, dos veces al dia. RISPERDAL es bien tolerado en este grupo de pacientes. Niños Se carece de experiencia en niños menores de 15 años. Entermedod renal o hepática Se recomienda iniciar el tratamiento con dosis de $0,5 \mathrm{mg}$, dos veces al día. Esta dosis puede individualizarse mediante incrementos de $0,5 \mathrm{mg}$. 2 veces al dia, hasta $1.2 \mathrm{mg}$ dos veces al día. RISPERDAL debe usarse con precaución en este grupo de pacientes hasta que se posea más experiencia. c) Contraindicaciones RISPERDAL está contraindicado en pacientes con conocida hipersensibilidad al producto. d) Advertencias y precauciones especiales de empleo Debido a la actividad $a$-bloqueadora de RISPERDAL puede manifestarse hipotensión ortostática, especiolmente durante el periodo inicial de aiuste de la dosis. RISPERDAL debe usarse con precaución en pacientes con enfermedades cardiovasculares ( $p . e$. insuficiencia cardiaca, infarto de miocardio, alteraciones de la conducción, deshidratación, hipovolemia o enfermedad cerebrovascular) y seguir las recomendaciones de ajuste gradual de la dosis (ver Posologia y método de administración). Si se manifestase hipotensión debe considerarse una reducción de la dosis. Los fármacos poseedores de propiedades antagonistas del receptor de la dopamina han sido relacionados con la inducción de discinesia tardia, la cual se caracteriza por movimientos rítmicos involuntarios, fundamentalmente de la lengua y/o cara. Se ha comunicado la aparición de sintomas extrapiramidales como un posible fac tor de riesgo en el desarrollo de la discinesia tardia. Dado que el potencial de RISPERDAL para inducir síntomas extrapiramidales es más bajo que el de los neurolépticos clásicos, el riesgo de inducir discinesia tardía se reduce en comparoción con estos últimos. Si aparecen signos y síntomas de discinesia tardía deberá considerarse la suspensión del tratamiento con todos los fármacos antipsicóticos. Con los neurolépticos clásicos se ha informado del llamado Sindrome Maligno de los Neurolépticos, caracterizado por hipertermia, gidez muscular, inestabilidad automática, alteraciones de la conciencia y aumento de los niveles de CPK. En caso de producirse deberá suspenderse el tratamiento de todos los fármacos antipsicóticos incluido RISPERDAL y se enviará urgentemente al paciente a un centro hospitalario. En caso de pacientes geriátricoso afectos de insuficiencia renal o hepática se recomienda disminuir a la mitad tanto la dosis inicial como los posteriores incrementos de dosis. RISPERDAL debe prescribirse con precaución a pacientes con parkinson ya que, teóricamente, puede causar un empeoramiento de esta enfermedad. También es conocido que los neurolépticos clósicos disminuyen el umbral de convulsiones. Se recomienda prudencia cuando se trate a pacientes epilépticos. Debe aconsejarse a los pacientes que se abstengan de realizar comidas excesivas, dada la posibilidad de aumento de peso. Risperdal comprimidos contienen lactosa. Se han descrito casos de intolerancia a este componente en niños y adolescentes. Aunque la cantidad presente en este preparado no es probablemente, suficiente para desencadenar los sintomas de intolerancia, en caso de que apareciesen diarreas debe de consultar a su médico. $\theta$ ) interacciones con otros medicamentos y otras formas de interacción No se han evaluodo de forma sistemática los riesgos de utilizar RISPERDAL en combinación con otros fármacos. Dados los efectos básicos de RISPERDAL sobre el SNC debe usarse con precaución cuando se utilice en combinación con otros fármacos de acción central. RISPERDAL puede antagonizar el efecto de la levodopa asi como de otros ogonistas de la dopamina. Se ha demostrado que la carbamacepina disminuye los niveles plasmáticos de la fracción antipsicótica activa de RISPERDAL. Se han observado efectos similares con otros inductores de los enzimas hepáticos. En la interrupción de carbamacepina otros inductores enzimáticos hepáticos se debe de reevaluar la dosis de RISPERDAL y si es necesario disminuirla. Fenotiazinas, antidepresivos triciclicos y algunos betabloqueantes pueden aumentar las concentraciones plasmáticas de risperidona pero no la fracción antipsicotica. Cuando RISPERDAL se administra conjuntamente con otros fármacos de fuerte unión a proteinas plasmáticas no se produce un desplazamiento significativo de ninguno de los fármacos de las mis mas. Los alimentos no afectan la absorción de RISPERDAL. D Embarazo y lactancia Embarazo: Categoría B3. No se ha comprobado la seguridad de RISPERDAL durante el embarazo en humanos. Aunque, en animales de experimentación, risperidona no ha mostrado toxicidad directa sobre la función reproductora, se han observado ciertos efectos indirectos mediodos por prolactina y Sistema Nervioso Central. No se ha detectado ningún efecto teratogénico en ningún estudio. No obstante, sólo debe usarse RISPERDAL durante el embarazo cuando los beneficios justifican los riesgos. Se desconoce si RISPERDAL se excreta por la leche humana. En estudios en animales, risperidona y 9-hidroxi-risperidona se excretan por g) Electos sobre la capacidad de conducir y utilizar maquinaria RISPERDAL pue

de interferir con aquellas actividades que requieran alerta mental. Consecuentemente, debe aconsejarse

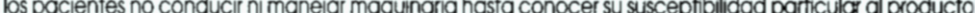
h) Reacciones adversas Basándose en una experiencia clínica muy amplia que incluye tratamiento de lar ga duración, RISPERDAL es generalmente bien tolerado. RISPERDAL es generalmente bien tolerado y en lo mayoría de los casos resulta dificil diferenciar los efectos adversos de los sintomas de la enfermedad subya-

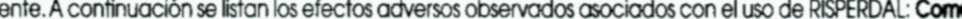
nes Insomnio, agitación, ansiedad, jaqueca. Menos comunes Somnolencia fatiga, mareos, falta de concentración, estreñimiento, dispepsia, náuseas/vómitos, dolor abdominal, visión borrosa, priapismo, disfunción de la erección, de la eyaculación y orgásmica, incontinencia urinaria, rinitis, rash y otras reacciones alérgicas. RISPERDAL posee una menor tendencia que los neurolépticos clásicos a inducir síntomas extrapiramidales. No obstante, en algunos casos, pueden manifestarse los siguientes sintomas: temblores, rigidez, hipersalivación, bradicinesia, acatisia, distonia aguda. Estos sintomas son habitualmente leves y reversibles al disminuir la dosis y/o al administrar, en caso necesario medicación antiparkinsoniana. Ocasionalmente, durante el tratomiento con RISPERDAL se han observodo hipotensión (ortostática), taquicardia (refleja) o hipertensión ver precauciones). Se ha informado de una disminución ligera del recuento de neutrofilos o/y trombocitos. SPERDAL puede inducir un aumento dosis-dependiente de las concentraciones plasmáticas de prolactina. por lo cual pueden manifestarse galactorrea, ginecomastia, alteraciones del ciclo menstrual y amenorreas. Durante el tratamiento con RISPERDAL se ha observado aumento de peso, edema y aumento de los niveles de enzimas hepáticos. Como los neurolépticos clásicos, ocasionalmente se ha informado de los siguientes efectos en pacientes psicóticos: intoxicación acuosa debido o bien a polidipsia psicogénica bien al síndrome de secreción inapropioda de la hormona antidiurética (SIADH), discinesia tardía, síndrome neuroléptico maligno, falta de regulación de la temperatura corporal y convulsiones. i) Sobredosificación Síntomas En general, los síntomas comunicados son aquellos resultantes de una exageración de los efectos armacológicos conocidos del producto incluyendo mareos, sedación, taquicardia, hipotensión y síntoma: extrapiramidales. Se ha comunicado sobredosificación por encima de $360 \mathrm{mg}$. Los datos de que se disponen sugieren un amplio margen de seguridad. En un paciente con hipocaliemia concomitante que tom $360 \mathrm{mg}$, se comunicó un prolongamiento del intervalo Q. En caso de sobredosificación aguda debe tene se en cuenta la posible implicación de polimedicación. Iratamiento Deben mantenerse libres las vías respirotorias y asegurar una ventilación y oxigenación adecuadas. Debe considerarse la posibilidad de lavaco gástrico (tras intubación si el paciente está inconsciente) y administración del carbón activo conjuntamente con un laxante. Debe establecerse inmediatamente monitorización cardiaca, la cual incluirá monitoriza ción electracardiográfica continua con el fin de detectar posibles arritmias. No existen antidotos específicos para RISPERDAL. Consecuentemente, deberán instituirse las medidas de soporte adecuadas. La hipotensión y el colapso respiratorio deberán tratarse con medidas adecuadas tales como fuidos intravenosos y agentes simpaticomiméticos. En caso de presentarse sintomas extrapiramidales graves, se administraráunc medicación anticolinérgica. Debe mantenerse monitorizoción interna y supervisión médica hasta que el paciente se recupere. 5. Propiedades farmacológicas a) Propiedades formacodinámicas RISPERDAL es un antagonista monoaminérgico selectivo con propiedades únicas. Posee una alta afínidad por los receptores 5-HT, serotoninérgicos y $D_{2}$ dopaminérgicos. RISPERDAL se une también a los receptores $\alpha$-adrenérgicos, y con baja actividad a los receptores $\mathrm{H}_{1}$-histaminérgicos y $\alpha_{2}$-adrenérgicos. Risperidona no tiene afinidad por los receptores colinérgicos. Aunque RISPERDAL es un potente antagonista $\mathrm{D}_{2}$ lo cual se considera que mejode catalepsia que los neurolépticos clásicos. El equilibrado antagonismo central de serotonina y dopaminc puede disminuir el riesgo de efectos secundarios extrapiramidales y extender su actividad terapéutica a los sintomas negativos y afectivos de la esquizofrenia. b) Propiedades larmacocinéficas RISPERDAL se absorbe completamente tras su administración por via oral, alcanzando concentraciones plasmáticas máximas entre 1 y 2 horas. Los alimentos no atectan a su absorción, por lo cual, RISPERDAL puede administrarse con o sin comidas. RISPERDAL se metaboliza parcialmente a 9-hidroxi-risperidon a la cual tiene una actividad farma cológica similar a la de risperidona. Risperidona y 9-hidroxi-risperidona forman pues la fracción antipsicótico activa. Otro via de metabolización de RISPERDAL es la N-desalquilación. Iros la administración oral a pacienes psicóticos, la risperidona se elimina con una semivida de alrededor de 3 horos. La semivida de elimina ción, tanto de la 9-hidroxi-risperidona como de la fracción antipsicótica activa es de 24 horas. El estado de equilibrio ("steady - state") de risperidona se alcanza, en la mayorío de los pacientes, durante el primer dí El estado de equilibrio de la 9-hidroxi-risperidona se alcanza dentro de los 4 ó 5 dias de tratamiento. Las concentraciones plasmáticas de RISPERDAL son proporcionales a la dosis administrada, dentro del rango de dosis terapéuticas establecido. RISPERDAL se distribuye rápidamente. El volumen de distribución es de 1-2 litros por kilo. En plasma RISPERDAL se une a la abúmina y a las $\alpha$-glucoproteinas ácidas L L u unión de risperidono a proteinas plasmáticas es del $88 \%$ y de la 9-hidroxi-risperidona del $77 \%$. Una semana después de su admini fración, el $70 \%$ de la dosis se ha eliminado por via urinaria y el $14 \%$ por las heces. En la orina, risperidona y hidroxi-risperidona representan el 35 - $45 \%$ de la dosis administrada. Los demás metabolitos son inactivos. Un ensayo de dosis única mostró mayores concentraciones plasmáticas activas y uno eliminación más lento de RISPERDAL en pacientes geriátricos y en pacientes con insuficiencia renal. La concentración plasmático de RISPERDAL es normal en pacientes con insuficiencia hepática. c) Datos prectínicos de seguridad No ho ningún hallazgo que comunicar que sea relevante y que no esté incluido en otras secciones. 6. Datos farmacéuticos a) Relaciones de excipientes Comprimidos: Núcleo del comprimido Lactosa Almidón de ma iz Celulosa microcristalina Hipromelosa $291015 \mathrm{mPa} . \mathrm{s}^{(0)}$ Estearato magnésico Silice anhidra coloidal Lauri. sulfato sódico Recubrimiento Hipromelosa 29105 mPa.s Propilen glicol Dióxido de titanio (E171) ${ }^{(0)}$ Talco Amarillo de quinoleina (E1OA) (a) (a) Sólo en comprimidos de 1 y $3 \mathrm{mg}$. Sólo en comprimidos de $3 \mathrm{mg}$. Solv. ción oral: Ácido tartárico Ácido benzoico Hidróxido sódico Agua purificada b) incompatibllidades La solu ción oral de RISPERDAL es incompatible con el té. c) Período de validez RISPERDAL 1 mg y RISPERDAL $3 \mathrm{mg}$ comprimidos: 3 años en todas las zonas climáticas cuando se acondiciona en blisters de PVC.PE-PVDC/A RISPERDAL solución oral ( $1 \mathrm{mg} / \mathrm{ml}): 2$ años para todas las zonas climáticas protegido de la congelación. Precauciones especiales de conservación RISPERDAL comprimidos se almacenará entre $15^{\circ} \mathrm{C}$ y $30^{\circ} \mathrm{C}$. RISPERDAL solución oral se almacenará entre $15^{\circ} \mathrm{C}$ y $30^{\circ} \mathrm{C}$ y se protegerá de la congelación. Mantener fue del alcance de los niños. $\theta$ ) Naturaleza y contenido del recipiente Los comprimidos se acondicionan en bilister PVC-PE-PVDC/Al consistentes en un a hoja de aluminio de 20 um con un recubrimiento de sellado térmico de $6 \mathrm{~g} / \mathrm{m}^{2}$ y una hoja de tres capas: PVC $200 \mu \mathrm{m}$, LDPE $25 \mu \mathrm{m}$, PVDC $90 \mathrm{~g} / \mathrm{m}^{2}$ Cada blister se acondiciona en una caja de cartón ( 20 o blister por caja). La solución oral se envasa en un frasco de vidrio topacio con cierre de piástico a prueba de niños. RISPERDAL solución oral de l $\mathrm{mg} / \mathrm{ml}$ se suministra en froscos de $100 \mathrm{ml}$ y $30 \mathrm{ml}$ con una jeringuilla de $3 \mathrm{ml}$, calibrada en miligramos y mililitros. El volumen mínimo es de $0,25 \mathrm{ml}$. El volumen máximo es de $3 \mathrm{ml}$. I) Nombre o razón social del fifular de comercialización: Janssen-Cilag, S.A. Paseo de Las Doce Estrellas, 5-7.28042 Madrid 7. Condiciones de prestación Farmacéutica del S.N.S. Con receta médica. A estas especialidades les corresponden APORTACION REDUCIDA (CICERO) 8. Presentaciones y P.V.P. (IVA4) 20 comprimidos

JANSSEN-CILAG, S.A. Paseo de las Doce Estrellas, 5 - 7 28042 - Madrid
$3 \mathrm{mg}: 9.251$ Ptas. N.M.; 60 comprimidos $3 \mathrm{mg}: 27.570$ Ptas. N.M.; $30 \mathrm{ml} 1 \mathrm{mg} / \mathrm{m}$ 4.681 Ptas. N.M.; $100 \mathrm{ml} 1 \mathrm{mg} / \mathrm{ml}$ : 15.601 Ptas. N.M. Texto Revisado: Jullo, 1998 


\section{EUROPEAN PSYCHIATRY}

Indexada en: BIOSIS/Biological Abstracts, Current Contents/Clinical Medicine and Social and Behavioural Sciences, EMBASE/Excerpta Medica, MEDLINE/Index Medicus, PASCAL/INIST-CNRS, Psychological Abstracts, Research Alert, Science Citation Index, SciSearch.

Editors-in-chief: P Boyer, Y. Lecrubier (Paris).

Secretariat: Hôpital de la Salpêtrière, INSERM U 302, Pavillon Clérambault, 47, bd de l'Hôpital, 75651 Paris cedex 13, France.

Tel: (33) 0142161656 . Fax: (33) 0145852800

Editors: JD Guelfi (Paris/Villejuif), R. Murray (London), M Maj (Naples), CB Pull (Luxembourg), M. Ackenheil (Munich), P Bech (Copenhagen)

Editors Emeritus: C Ballus (Barcelona), H Heimann (Tübingen)

Consejeros estadísticos: A. Heyting (Damarken), N. Takei (Hamamatso), B. Fallisord (Paris).

\section{Consejo Editorial}

J. Adès, Colombes, France

H.S. Akiskal, Rockville, MD, USA

N.C. Andreasen, Iowa City, IA, USA

J. Angst, Zurich, Switzerland

M. Ansseau, Liège, Belgium

P. Baumann, Lausanne, Switzerland

H. Beckmann, Würzburg, Germany

P. Berner, Vienna, Austria

A. Bertelsen, Risskov, Denmark

J. Biber, Cadiz, Spain

J. Birley, London, $U K$

J.C. Bisserbe, Meudon, France

B. Bondy, Munich, Germany

J.P. Boulenger, Sherbrooke, Canada

M. Bourgeois, Bordeaux, France

F. Brambilla, Milan, Italy

I. Brockington, Birmingham, UK

A. Clare, Dublin, Ireland

F. Clerget-Darpoux, Paris, France

V. Conde Lopez, Valladolid, Spain

S. Consoli, Paris, France

P. Cosyns, Antwerp, Belgium

J. Cottraux, Lyon France

M. von Cranach, Kaufbeuren, Germany

A. Dahl, Oslo, Norway

J.M. Danion, Strasbourg, France

J.F.W. Deakin, Manchester, UK

M. de Bonis, Le Kremlin Bicêtre, Paris, France

H. Dufour, Lausanne, Switzerland

R. Engel, Munich, Germany

T. Fahy, Galway, Ireland

L. Farde, Stockholm, Sweden

A. Féline, Le Kremlin Bicêtre, Paris, France

A. Fernandes da Fonseca, Porto, Portugal

F. Ferrero, Geneva, Switzerland

\section{Fichter, Prien am Chiemsee, Germany}

H. Freeman, London, UK

H.J. Gaertner, Tiibingen, Germany

D. Goldberg, Manchester, UK

I. Hand, Hamburg, Germany

H. Häfner, Mannheim, Germany

T. Helgason, Reykjavik, Iceland

H. Hippius, Munich, Germany

A. Jablenski, Sofia, Bulgaria

E. Johnston, Edinburgh, $U K$

S. Kasper, Vienna, Austria

M. Kastrup, Hvidovre, Denmark

D. Kemali, Naples, Italy

R. Kendell, Edinburgh, UK

D. Klein, New York, NY, USA

R. Klein, New York, NY, USA

S. Langer, Paris, France

J. Lellouch, Villejuif, France

P. Lemoine, Lyon, France

T. Lemperière, Colombes, France

J.P. Lépine, Paris, France

O.M. Lesch, Vienna, Austria

S.W. Lewis, London, UK

H. Lôo, París, France

J.J. López-Ibor, Madrid, Spain

P. McGuffin, Cardiff, $U K$

W. Maier, Mainz, Germany

A. Mann, London, UK

K. Mann, Tübingen, Germany

I. Marks, London, UK

J. Marlet, Venray, The Netherlands

J. Massanna, Barcelona, Spain

J. Mendlewicz, Brussels, Belgium

H.J. Möller, Munich, Germany

N. Müller, Munich, Germany
M. Musalek, Vienna, Austria

D. Naber, Munich, Germany

E. O'Callaghan, Dublin, Ireland

Y. Ono, Tokyo, Japan

M. Patris, Strasbourg, France

J. Pellet, Saint-Etienne, France

C. Perris, Umeä, Sweden

P. Pichot, Paris, France

T. Pohlmächer, Munich, Germany

H. Pope, Belmont, MA, USA

A.J. Puech, Paris, France

G. Racagni, Milan, Italy

N. Retterstøl, Oslo, Norway

M.A. Ron, London, UK

R. Rosenberg, Risskov, Denmark

M. Roth, Cambridge, UK

F. Rouillon, Colombes, France

J. Saiz-Ruiz, Madrid, Spain

A. Sánchez-Blanque, Zaragoza, Spain

N. Sartorius, Geneva, Switzerland

F. Schulsinger, Copenhagen, Denmark

G. Sedvall, Stockholm, Sweden

L. Singer, Strasbourg, France

C.N. Stefanis, Athens, Greece

E. Straube, Tübingen, Germany

E. Taylor, London, $U K$

P. Taylor, London, $U K$

L. Träskman-Bendz, Lund, Sweden

J. Vallejo, Barcelona, spain

L. Waintraub, Paris, France

D. Widlöcher, Paris, France

J. Wilmotte, Charleroi, Belgium

J. Wing, London, UK

F.T. Zimmer, Tübingen, Germany

J. Zohar, Beer-Sheva, Israel

\section{Association of European Psychiatrists}

President: J Angst (Zürich); Past President: R Murray (London); President Elect: N Sartorius (Geneva); Secretary General: CB Pull (Luxembourg); Treasurer: M Patris (Strasbourg); Counsellors: H Häfner (Mannheim), Y Lecrubier (Paris); Section: L Singer (Strasbourg).

European Psychiatry, edición original, es publicada por Editions Scientifiques Elsevier; 23 rue Linois, 75724 París cedex 15, Francia.

Director de la Edición Española: C. Ballús

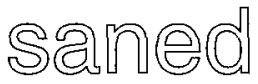

SANIDAD EDICIONES

Sanidad y Ediciones (SANED, S.L.). C/ Capitán Haya, 60. 28020 MADRID. Telf.: (91) 74995 06. saned@medynet.com

Caspe, 172, 4. ${ }^{\circ}$-A. BARCELONA. Telf.: (93) 2472411

Directora Editorial: Alicia Martínez Magro.

Suscripciones: SANED, S.L. Capitán Haya, 60. 28020 MADRID.

Publicación Bimensual ( 8 números al año).

Composición: Artecomp, S.L. Albarracín, 50-1.. Madrid. Imprime: D.G.B. Resina, 13-15. Madrid.

Soporte válido M. Sanidad: SV 93040 R. ISSN: 1134-0665. Depósito Legal: M-5991-1994.

Copyright de los textos originales 1999. Reservados todos los derechos. Ninguna parte de esta publicación puede ser reproducida, transmitida en ninguna forma o medio alguno, electrónico o mecánico, incluyendo fotocopias, grabaciones o cualquier sistema de recuperación de almacenaje de información, sin la autorización por escrito de los titulares del Copyright. 


\section{EUROPEAN PSYCHIATRY \\ EDICIÓN ESPAÑOLA - REVISTA DE LA ASOCIACIÓN EUROPEA DE PSIQUIATRIA}

\section{Sumario Vol. 6 - Núm. 6 - Septiembre 1999}

\section{ARTÍCULOS ORIGINALES \\ Comportamiento automutilador de pacientes psiquiátricos hospitalizados}

H. L. I. Nijman, M. Dautzenberg. H. L. G. J. Merckelbach, P. Jung,

I. Wessel, J. à Campo . . . . . . . . . . . . . . . . . . .

Detección de la ansiedad y la depresión en pacientes mayores hospitalizados utilizando un inventario breve

P. Huber, R. Mulligan, A. Mackinnon, T. Nebuloni-French,

J. P. Michel . .

Uso de ansiolíticos e hipnóticos en 376 pacientes psiquiátricos hospitalizados

P. Hardy, C. Payan, J. C. Bisserbe, J. P. Lepine y Grupo

de Neuopsicotropos GERMED .

Una comparación doble ciego de sertralina y fluoxetina en el tratamiento de episodio depresivo mayor en pacientes ambulatorios

D. Sechter, S. Troy, S. Paternetti, P. Boyer . . . . . . . . . . .

\section{PERSPECTIVAS}

Estadios de adaptación a un diagnóstico médico de una condición somática grave

J. E. Mękarski.

\section{COMUNICACIÓN BREVE}

\section{Anomalías en el movimiento ocular sacádico en la} demencia

H. J. Schewe, R. Uebelhack, K. Vohs .
Dumirox $50 \mathrm{mg}$ - Dumirox $100 \mathrm{mg}$ COMPOSICION CUALITATIVA Y CUANTITATIVA: Principio activo: Fluvoxamina (DCl) maleato. Cada comprimido recubierto contiene 50 o $100 \mathrm{mg}$ de maleato de fiuvoxamina FORMA FARMACEUTICA: Dumirox $50 \mathrm{mg}$ : comprimidos recubiertos de color blanco con la inscripción "duphar" en una de sus caras y "291" en la otra. Dumirox $100 \mathrm{mg}$ : comprimidos recubiertos y ranurados. de color blanco con la inscripcion "duphar" en una de sus caras y " $313^{\prime \prime}$ en la otra. DATOS CLínICOS: Indicaciones terapéuticas. Tratamiento de la enfermedad depresiva y de los sintomas de los trastornos depresivos. Tratamiento de los sintomas del trastorno obsesivo compuisivo. Posologia y forma de administracion. Depresion: La dosis inicial recomendada es de 50 a $150 \mathrm{mg}$. administrada como dosis unica, preferentemente por la noche Se recomienda incremertar la dosis de un modo gradual hasta alcanzar re segin la respuesta la dosis considerada como eficaz. La dosis media eficaz es de $100 \mathrm{mg}$ y debe ajustarse segun la respuesta Individual de cada paciente. Se han administrado dosis de hasta $300 \mathrm{mg}$ por dia. Si la dosis total diaria supera
los $150 \mathrm{mg}$. es aconsejable que esta sea dividida en varias tomas. De acuerdo con las normas establecidas los $150 \mathrm{mg}$, es aconsejable que esta sea dividida en varias tomas. De acuerdo con las normas establecidas por la OMS, debe continuarse la medicación antidepresiva como minimo durante 6 meses despues de superar
el episodio depresivo. Trastorno obsesivo compulsivo: La dosis eficaz habitual es entre $100 \mathrm{mg}$ y $200 \mathrm{mg}$ aunque algunos pacientes pueden necesitar hasta $300 \mathrm{mg}$ diarıos. Se recomienda una dosis inicial de $50 \mathrm{mg}$ por dia durante 304 dias. La cosis debera incrementarse progresivamente hasta aicanzar la dosis eficaz. con un máximo de $300 \mathrm{mg}$ diarios. Las dosis de hasta $150 \mathrm{mg}$ pueden administrarse como una dosis única, preferentemente por la noche. Si la dosis total diaria supera los $150 \mathrm{mg}$. es aconsejable que ésta sea dividida en 2 o 3 tomas separadas. Si al cabo de 10 semanas no se observa mejoria. debe reconsiderarse el tratamiento con fluyoxamina. Aunque hasta la fecha no existen estudios sistemáticos que respondan a ia

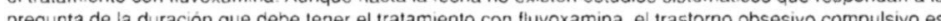
pregunta de la duracion que debe tener el tratamiento con fluvoxamina. el trastorno obsesivo compulsivo es una condicion cronica. y parece razonable considerar la continuacion del tralamiento mas alla de las de la dosis en base al paciente, manteniendolo con la dosis minima eficaz. La necesidad de tratamiento farmacoiógico debera reevaluarse periódicamente. Algunos psiquiatras defienden la asociación con terapia conductuai en aquelios pacientes que han respondido al tratamiento farmacológico. Los pacientes con insuficiencia hepática o renal deberán iniciar el tratamiento con una dosis baja y serán cuidadosamente monitorizados por su medico. Los comprimidos deben tragarse con ayuda de un poco de agua, sin masticar. Contraindicaciones: No puede administrarse conjuntamente con inhibidores de la M.A.O. Puede iniciarse tratamiento con fluvoxamina dos semanas despues de finalizar un tratamiento con un inhibidor irreversible de la M.A.O. o el dia siguiente de finalizar un tratamiento con un inhibidor reversible de la M. A.O. Debe esperarse al menos una semana entre la interrupción de la medicación con Dumirox ${ }^{*}$ y la adminstración esperaliquer inhibidor ce $\mathrm{MAO}$ Contraind cado en caso de hipersensiblidad a cualquiera de los componentes Advertencias y precauciones especiales de empleo: La posibilidad de tentativas de suicidio inherente los entermos depresivos puede persistir hasta que no se produzca una mejoria significativa. Los pacientes los entermos depresivos puede persistir hasta que no se produzca una mejoria significativa. Los pacientes
con insuficiencia hepatica o renal deberan iniciar el tratamiento con una dosis baja y serán cuidadosamente con insuficiencia hepática o renal deberan iniciar el tratamiento con una dosis baja y serán cuidadosamente monitorizados por su medico. Ocasionalmente. el tratamiento se ha visto asociado a una elevación de los enzimas hepáticos, a veces acompañándose de sintomas. En estos casos. se recomienda interrumpir la medicación. Aunque en los estudios en animales fluvoxamina no ha evidenciado poseer actividad proconvulsiva. es aconsejable tener precaucion al administrar el fármaco a pacientes con antecedentes de episodios convulsivos. Si aparecen convulsiones, el tratamiento debe interrumpirse. Los datos de que se dispone no parecen mostrar diferencias farmacocinéticas significativas entre los individuos jovenes y ancianos. Sin embargo, y de acuerdo a estos estudios. los incrementos de dosis deberan realizarse con precaución de forma más lenta en los pacientes ancianos. Dumirox puede producir una ligera disminucion de la frecuencia cardiaca (2-6 latidos por minuto). Debido a la falta de experiencia se desaconseja su empleo en niños. Interacción con otros medicamentos y otras formas de interaccion: No administrar con inhibidores de la M.A.O. (ver contraindicaciones). Fluvoxamina puede prolongar la eliminación de farmacos que se metabolicen por oxidación en el higado. Es conveniente tener en cuenta la posibilidad de interaccion con fármacos de margen terapeutico estrecho (por ejemplo, warfarina. fenitoina. teofilina y carbamacepina). Es probable que puedan incrementarse los niveles plasmáticos de benzodiacepinas con metabolismo oxidativo durante el tratamiento concomitante con fluvoxamina. Se ha descrito una elevación de los niveles plasmáticos de antidepresivos triciclicos al ser administrados junto con fluvoxamina. No es. por tanto. aconsejable 1 combinación de estos farmacos. En diversos estudios de interaccion se han observado elevaciones de los niveles plasmaticos de propranolol durante la administracion concomitante con fluvoxamina. Por esta razon. se debera disminuir la dosis de este fármaco cuando se administre junto con Dumirox. Al administrar se debera disminir la dosis de este ármaco cuando se admistre junto con Dumirox-A adminicos de warfarina aumentaban significativamente. prolongándose también el tiempo de protrombina. Por este motivo. warfarina aumentaban significativamente. prolongandose lambien el tiempo de protrombina. Por este motivo se recomienda monitorizar a los pacientes que tomen fluvoxamina y anticoagulantes orales por lo que respecta
al tiempo de protrombina y ajustar debidamente las dosis. No se ha observado interaccion con atenolol o al tiempo de protrombina y ajustar debidamente las dosis. No se ha observado interaccion con atenolol digoxina. Fluvoxamina ha sido utilizada en combinacion con ittio para el tratamiento de pacientes con depresion grave que han mostrado ser resistentes a medicacion Sin embargo. el itio (y posiblemente el hiplofanos incrementan los efectos serotoninergicos de lluvoxamina. por lo cual su utilizacion conjunta debera hacerse y precaucion. Se recomit. En los estudios de reproduccion en animales no se ha observado evidencia de y lactancia: che lerilidad. (a) Fluvoxamina se excreta por la leche materna en pequenas cantidades, por lo cual no debe ser administrado fluvoxamite la lactancia. Efectos sobre la capacidad de conducir y manejar maquinaria: En dosis de hasta durante la lactancia. Efectos sobre la capacidad de conducir y manejar maquinaria: En dosis de hasta
$150 \mathrm{mg}$. fluvoxamina no parece afectar la capacidad psicomotriz asociada a la habilidad de conduccion $150 \mathrm{mg}$. fluvoxamina no parece afectar la capacidad psicomotriz asociada a la habilidad de conduccion
manejo de maquinaria complicada en voluntarios sanos. No obstante, se tendra precaución hasta que la respuesta a la medicacion haya sido establecida. Reacciones adversas: Las reacciones adversas más Este efecto suele desaparecer durante las dos primeras semanas de tratamiento. Otras reacciones descritas en los estudios controlados y con una trecuencia superior al $1 \%$ son. Sistema nervioso central: somnolencia mareos. cefalea. insomnio. nerviosismo. agitación, ansiedad. temblor. Aparato digestivo: constipación. anorexia dispepsia. diarrea, malestar abdominal boca seca. Aparato urogental: alteraciones de la eyaculacion (eyaculacion retardada) Piel sudoracion Otros: astena Algunas de las reacciones reportadas pueden ser sintomas de 1 entermedad depresiva y no ser. por consiguiente, inducidas por la med cacion. Sobredosificacion: Sintomas Los sintomas mas comunes incluyen trastornos gastrointestinales (náuseas. vomitos y diarrea), somnolencia

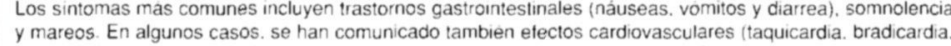
y mareos. En algunos casos. se han comunicado tambien efectos cardiovasculares (taquicardia. bradicardia.
hipotension). trastornos de la funcion hepatica. convuisiones y coma. Hasta la fecha. han sido comunicados màs de 300 casos de sobredosificacion deliberada con fluvoxamina. La dosis máxima que se conoce ingerida por un paciente son $10.000 \mathrm{mg}$. Dicho paciente se recuperó totalmente instaurando solo un tratamiento sintomatico. Ocasionalmente. se han registrado casos de sobredositicacion mas graves con fluvoxamina en combinación con otros tảrmacos. Solo en dos casos se han producido muertes por sobredosis con fluvoxamina exclusivamente. Tratamiento No se dispone de antidoto especifico En caso de ingesta masiva accidental se procedera a lavado de estomago y se instaurara un tratamiento sintomatico. Se recomienda el tratamiento con carbón activado Es poco probable que la diuresis torzada o dialisis puedan resultar beneficiosas. OATOS FARMACEUTICOS: Relacion de excipientes: Maniz. Almidon de maz. Almidón pregelatinizado Esteaniltumarato sodico. Silice coloidal anhidra. Metilhidroxipropilcelulosa. Polietilenglicol 6000 Talco y Dioxido de titanio Incompatibilidades: No procede. Periodo de validez: 3 anos en su acondicionamiento final. Precauciones Incompatibilidades: No procede. Periodo de validez: 3 años en su acondicionamiento final Precauciones
especiales de conservacion: Normales. Naturaleza y contenido del recipiente: Blisters de PVC PVDCaluminio. Cada estuche contiene Dumirox $50 \mathrm{mg} 30$ comprimidu. Dumirox $100 \mathrm{mg} 20$ comprimidos Instrucciones de uso y manipulacion: No procede Nombre o razon social y domicilio permanente de titular de la autorizacion: Duphar Nezel, S L. Avda. Diagonal. 507.08029 Barcelona. CONDICIONES DE PRESTACION: Reembolsable por el Sistema Nacional de Salud. Aportacion reducida Dumirox S0: PVP (IVA4): 1.178 ptas Dumirox 100: PVP. (IVA4 - 2029 plas

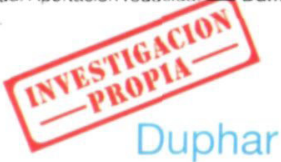
\&Upjohn 


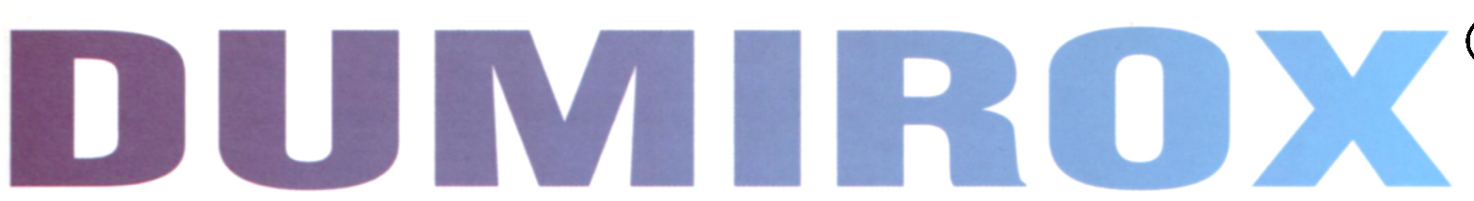

(R)

Fluvoxamina
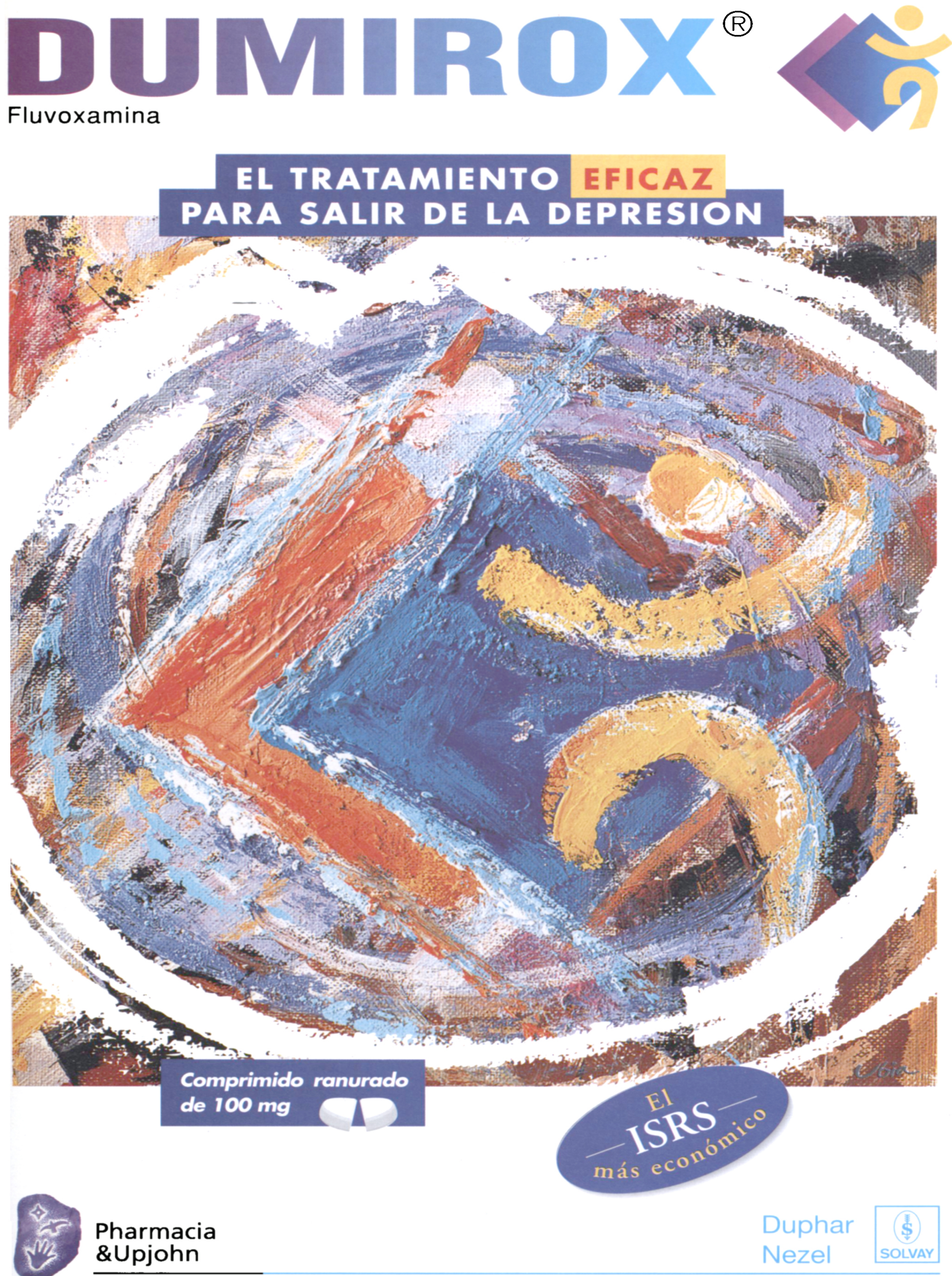


\section{EUROPEAN PSYCHIATRY}

EDICIÓN ESPAÑOLA - REVISTA DE LA ASOCIACIÓN EUROPEA DE PSIQUIATRIA

\section{Summary}

Vol. 6 - No. 6 - September 1999

\section{ORIGINAL ARTICLES}

Self-mutilating behaviour of psychiatric in patients

H. L. I. Nijman, M. Dautzenberg, H. L. G. J. Merckelbach, P. Jung,

I. Wessel, J. à Campo . . . . . . . . . . . . . . . . .

Detecting anxiety and depression in hospitalised elderly patients using a brief inventory

P. Huber, R. Mulligan, A. Mackinnon, T. Nebuloni-French,

J. P. Michel.

Anxiolytic and hypnotic use in $\mathbf{3 7 6}$ psychiatric in patients

P. Hardy, C. Payan, J. C. Bisserbe, J. P. Lepine and GERMED

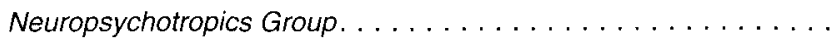

\section{A double-blind comparison of sertraline and fluoxetine in} the treatment of major depressive episode in outpatients

D. Sechter, S. Troy, S. Paternetti, P. Boyer . . . . . . . . . . .

\section{PERSPECTIVES}

\section{Stages of adjustment to a medical diagnosis of a serious somatic} condition

J. E. Mękarski.

SHORT COMMUNICATION

Abnormality in saccadic eye movement in dementia

H. J. Schewe, R. Uebelhack, K. Vohs . . . . . . . . . . . .
NOMBRE DEL MEDICAMENTO: PRISDAL $20 \mathrm{mg}$ Comprimidos. COMPOSICIÓN CUALITATIVAY Y CUANTITATIVA: Cada comprimido contiene: Cltalopram (DCli) (bromhidrato) $20 \mathrm{mg}$, excipientes
(contenido lactosa), C.S. FORMA FARMACEUTICA: Comprimidos recubiertos. DATOS CLiNiCOS: Indicaciones terapeuticas: Episodios depresivos mayores. Trastorno de angustia con o sin agoratobia. Posologia y forma de administracion: Este medicamento esta indicado exclusivamente para
el adulto y el anciano. Depresión: Adultos: La dosis minima eficaz es de $20 \mathrm{mg} / \mathrm{dia}$. Dependiendo

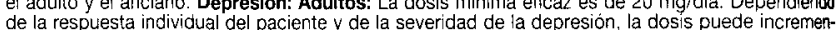

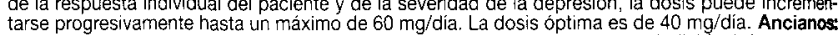
La dosis diaria recomendada es de $20 \mathrm{mg} / \mathrm{d}$ dia. Dependiendo de la respuesta individual del pacienta y de la severidad de la depresion, la dosis se puede incrementar hasta un máximo de $40 \mathrm{mg} / \mathrm{dia}$ Duración del tratamiento: El electo antidepresivo generalmente se inicia después de 2 a 4 sema-
nas de iniciado el tratamiento. El tratamiento con antidepresivos es sintomático y, por tanto, debe nas de iniciado el tratamiento. El tratamiento con antidepresivos es sintomatico y, por tanto, debe continuarse durante un periodo de tiempo apropiado, generalmente durante seis meses, a fin de
prevenir recaidas. No existen estudios sobre prevención de recurrencias. Trastorno de angustia: prevenir recaidas. No existen estudios sobre prevención de recurrencias. Trastorno de angustia:
Adultos: La dosis inicial es de $10 \mathrm{mg} /$ dia. Despues de una semana se incrementa la dosis a 20 $\mathrm{mg} / \mathrm{dia}$. La dosis optima es de $20-3 \mathrm{~m} \mathrm{~m} /$ dia. En caso de no obterer respuesta suficiente, esta dosis puede incrementarse hasta un maximo de ang/de incrementar la dosis a $20 \mathrm{mg} / d \dot{d}$. La dosis puede incrementarse hasta un maximo de $40 \mathrm{mg} / \mathrm{dia}$ dependiendo de la resp a 20 mg madaila. La dosis puede Duración del tratamiento: $\mathrm{El}$ tratamiento es de larça duración. Se ha comprobado que se mantiene el efecto terapéeutico en tratamientos a laroo piazo $\{1$ año). En caso de insuficiencia hepática: Los pacientes afectos de función hepática disminuida no deberian recibir dosis superiores a 30 $\mathrm{mg} / \mathrm{dia}$. En caso de insuficiencia renal: No se requieren precauciones especiales para los pacientes con insuficiencia renal leve o moderada. Hasta el momento no se dispone de información para el tratamiento de pacientes con función renal severamente disminuida (aclaramiento de la creatinina $<20 \mathrm{ml} / \mathrm{min}$ ). Forma de administración: Los comprimidos de citalopram pueden administrarse en una sola toma diaria, en cualquier momento del dia, independientemente de las comidas. creatinina inferior a 20 meninuto date la ausencia de datos. Niños de menos de 15 años de edad no se dispone de datos. Asociación con los IMAO no selectivos e IMAO selectivos B (víase interacciones con otros medicamentos). Contraindicaciones relativas: asociaciones con los IMAO selectives A. Advertencias y precauciones especiales de empleo: Advertencias: Con los iM todo tratamiento con antidepresivos, el riesgo de suicidio persiste en el periodo inicial dei tratamiento en los pacientes depresivos, porque la supresión de la irhibición psicomotora puede preceder a la acción antidepresiva propiamente dicha. Dado que al inicio del tratamiento aparecen insomnio o nerviosismo, se puede considerar una disminucion de la cosis o un tratamiento sedante asociado
hasta la mejoria del episodio depresivo. Algunos pacientes con trastorno de angustia pueden prehasta la mejoria del episodio depresivo. Algunos pacientes con trastorno de angustia pueden presentar una intensificación de su sintomatologia al inicio del tratamiento con antidepresivos. Este
aumento paradójico inicial de la ansiedad es más aparente en los primeros dias, desapareciendo al conthuar el frata hento, en el plazo de 2 semanas desde el inicio del mismo. Esta especialidad contiene lactosa. Se han descrito casos de intolerancia a este componente en niños y adolescentes. La mas de intolerancia. Precauciones especiales de empleo: En caso de insuficiencia hepatica. metabolismo puede enlentecerse por lo que se debera reducir la dosis a la mitad ivease Posolog y forma de administraciónn). En caso de crisis maniaca, cebe interrumpirse el tratamiento con citalo. pram y prescribirse un neuroleptico sedante. La asociación de un inhibidor de la recaptacion de serotonina con un IMAO selectivo A (véase Interacciones con otros medicamentos) sólo debe llevarse a cabo como ultimo recurso, es decir, en el $5 \%$ de las depresiones resistentes a los tratamientos ensayados con anterioridad: sucesión de dos antidepresivos de mecanismo diferente prescritos en monoterapia, asociacion de un antidepresivo con litio. Si estas tres tentativas fracasan, esta asociación puede considerarse como uit mo recurso, pero impone un seguimiento extremada-
mente riguroso del paciente, teniendo en cuenta el riesco de sindrome serotoninérgico" viéase mas adelante) al que esta expuesto. En los pacientes epilepticos o que tengan antecedentes de epilepadelante) al que esta expuesto. En los pacientes epilepticos o que tengan antecedentes de eplies
sia es prudente reforzar la vigiancia clinica y terapia electroconvulsiva. La aparición de crisis convulsivas impone la interrupción del tratamiento. Aunque no se ha observado ninguna interacción clara con el alconol, se recomienda limitar su consumo. Interacciones con otros medicamentos y otras formas de interacción: Interacciones con otros medicamentos. ASOCÁACO ES SONun descanso de dos semanas entre la retirada de un IMAO y el inicio del tratamiento con citaliopram. $y$ de al menos una semana entre la retirada de citalopram y el inicio de un tratamiento con un IMAO. Sindrome serotoninergico: La asociación de IMAO, selectivo o no, con las moléculas que ininiben la
recaptación de la serotonina puede ser el origen de un "sindrome serotoninérgico". El litio que recaptación de la serotonina puede ser el origen de un "sindrome serotoninérgico". El litio que
aumenta la neurotransmisión serotoninérgica puede igualmente provocar un sindrome serotoninéraumenta la neurotransmision serotoninergica, puede igualmente provocar un sindrome serotoniner-
gico con los inhibidores de la recaptación de la serotonina, pero de una manera mas atenuada. Dicho sindrome se manifiesta por la aparición (en alguin caso brusca), simultanea o sucesiva de un conjunto de sintomas que pueden hacer necesaria la hospitalización o incluso producir la muerte: Estos sintomas pueden ser: psiquicos (agitación, confusión, hipomania, ocasionalmente coma): motores (mioclonias, temblores. hiperreflexia, rigidez, hiperactividad); vegetativos (hipo o hipertensión, taquicardia, escaloffios, hipentemia, sudores); digestivos (diarreal. Para poder ser identificado rente, y todo ello en ausencia de un tratamiento neuroleptico recientemente asociado o de un aumento reciente de las dosis de un tratamiento neuroléptico asociado, teniendo en cuenta las similitudes clinicas con el sindrome mal igno de los neurolepticos. El estricto respeto de la posologia indi-
cada constituye un factor esencial en la prevención de la aparicion de este sindrome. + MAOU seleccada constituye un factor esencial en la prevencion de la aparicion de este sindrome.
tivo $B$ (selegilinal (por extrapolación a partir de la fluoxetinal. Riesgo de hipertensión paroxistica y de tivo $B$ (selegilinal (por extrapolación a partir de la fluoxetina). Riesgo de hipertension paroxistica y de
sintomas de vasoconstriccion periferica. Respetar un descanso de dos semanas entre la retirada del IMAO-B y el inicio del tratamiento con citalopram, y de al menos una sermana entre la retirada del selectivo A (moclobemióa, toloxatonas). Riesgo de aparición de un sindrome serotoninergico* (vease anteriormente). Si la asociación es verdaderamente necesaria, realizar una vigilancia clínica muY

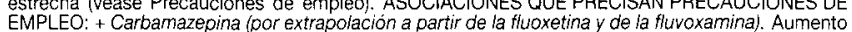
de los niveles de carbamazepina con signos de sobredosis. Vigilancia clínica con control de las concentraciones plasmáticas de carbamazepina y posible reducción de la posologia de carbamazepina durante el tratamiento con citalopram y después de su interrupción. + Litio (por extrapoiación a par-
tir de la fluoxetina y de la fluvoxaminal. Rieso de aparición de un sindrome serotoninérgico" véase
anteriormente). Vigilancia clinica regular. ASOCIACION A TENER EN CUENTA: I Imipramina. El citaanteriomente). Vigliancia clinica reguar. AS las concentraciones sanguineas de desipramina (metalopram comporta un aumento del
bolito principal de la imioramina). Embarazo y lactancia: La escasez de observaciones clinicas disponibles impone la prudencia en la mujer embarazada y durante la lactancia (veáse Schos precliricos de seguridad). Efectos sobre la capacidad para conducir vehiculos y utilizar maquinaria: recomendar prudencia a los conductores de vehículos y usuarios de máquinas. Reacciones adversas: Los efectos indeseables observados durante el tratamiento con citalopram son generalmente de intensidad leve o moderada. Serán evidentes durante la primera e incluso las dos primeras sema nas del tratamiento, y posteriormente desaparecen al mejorar el episodio depresivo. Se han encontrado, en monoterapia o en asociaciones con otros psicotropos, los siguientes trastomos. memoria, tendencia suicida; gastrointestinales: náuseas, vómitos, diarreas, estreñimiento, sequedad de boca; hepáticos: se han dado casos excepcionales de aumento de las enzimas hepáticas; cutáneos: exantema, prurito; visuales: trastornos de la adaptacion; metabolicos: perdida o aumento de
peso: cardiovasculares: taquicardia, hipotensión ortostatica, bradicardia en los pacientes que tienen una frecuencia cardiaca baja; de la libido; de la micción; diaforesis. Sobredosificación: Los principales sintomas hallados son; fatiga, vértigos, temblores de las manos, nauseas, somnolencia. el alcohol el citalasram no parece provocar a aparición de una sintomatologia clinica especifica. salvo con los IMAO selectivos A ivéase Interacciones con otros medicamentos y precauciones de empieol. El tratamiento es sintomatico, con lavado gasstrico lo antes posible tras la ingestión oral. La vigilancia médica deberá mantenerse durante al menos 24 horas. No existe tratamiento especifico. Datos preclínicos sobre seguridad: Citalopram tiene una toxicidad aguda débil. En los estudios de toxicidad crónica no se ha observado ningún elemento perjudicial en su empleo terapeutico. Durante los experimentos sobre animales, citalopram no se ha mostrado como teratogeno y no ha mocifi-
cado la gestación ni la mortalidad perinatal de las crias. Sin embargo, concentraciones muy bajas cado la gestación ni la mortalidad perinatal de las crias. Sin embargo, concentraciones masan a la leche (véase Embarazo y lactancia). Incompatibilidades: No se han descrito. Período de validez: 5 años. Precauciones especiales de conservación: Conservar a temperatura ambiente (inferior a $+25^{\circ} \mathrm{C}$ ). Naturaleza y contenido del recipiente: Envase conteniendo Ninguna Nombre y domicilio social del titular de la autorización de comercialización: ALMIRALL PRODESFARMA, S.A. General Mitre, 151 - 08022 Barcelona (España). P.V.P. (IVA): PRISOAL 14 comprimidos recubiertos, 2.723 ptas.: PRISDAL 28 comprimidos recubiertos, 5.446 ptas. Sistema Nacional de Salud. Aportación reducida. Fecha de elaboración: Enero 1998. 


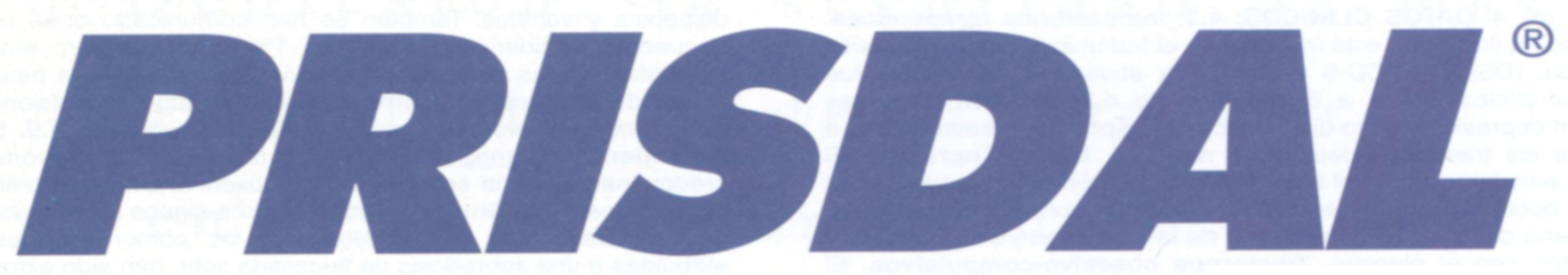

Citalopram

\section{Sin interferencias}

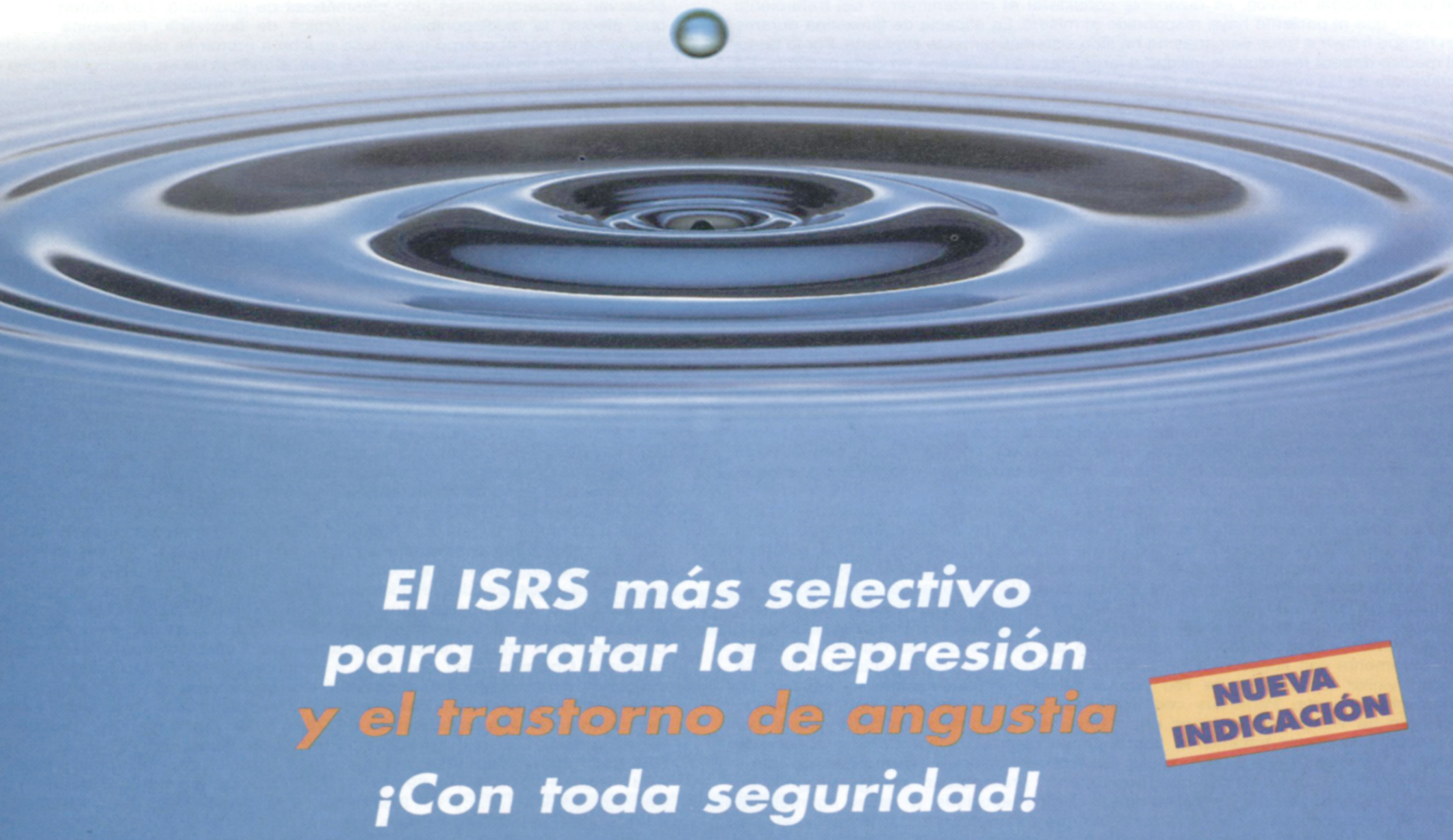


1. NOMBRE COMERCIAL: PROZAC $20 \mathrm{mg}$ Fluoxetina 2 COMPOSICIÓN: PROZAC $20 \mathrm{mg}$ cápsulas: cada cápsula contiene fluoxetina (D.C.I.) (clorhidrato) 20 $\mathrm{mg}$, excipientes c.s. PROZAC $20 \mathrm{mg}$ líquido: cada $5 \mathrm{ml}$ contienen fluoxetina (D.C.I.) (clorhidrato) $20 \mathrm{mg}$; sacarosa, aproximadamente $3 \mathrm{~g}$; otros excipientes c.s. PROZAC ${ }^{2}$ $20 \mathrm{mg}$ comprimidos: cada comprimido contiene fluoxetina (D.C.l.) (clorhidrato) $20 \mathrm{mg}$. Excipientes: sacarina $11,19 \mathrm{mg}$; otros excipientes, c.s. PROZAC ${ }^{\circledR} 20 \mathrm{mg}$ sobres: cada sobre contiene fluoxetina (D.C.I.) (clorhidrato) $20 \mathrm{mg}$. Excipientes: sacarina sódica 10 mg; otros excipientes c.s. 3. FORMA FARMACÉUTICA: Cápsulas, solución oral, comprimidos y polvo en sobres monodosis. El envase de la solución oral contiene un vasito dosificador de $5 \mathrm{ml}$. 4. DATOS CLÍNICOS: 4.1. Indicaciones terapéuticas. Depresión. El clorhidrato de fluoxetina está indicado en el tratamiento de la depresión y su ansiedad asociada. (DSM III, ICD-9 y RDC). La eficacia de fluoxetina fue establecida en ensayos clínicos de 5 a 6 semanas de duración con pacientes ambulatorios que sufrian depresión y cuyo diagnóstico correspondía estrechamente a la categoría DSM-III de los trastornos depresivos mayores. Bulimia nerviosa. El clorhidrato de fluoxetina está indicado en el tratamiento de la bulimia nerviosa. En dos ensayos controlados, a doble ciego y aleatorios, en pacientes con bulimia nerviosa, fluoxetina ha mostrado una disminución significativa de la voracidad y de la actividad purgante en comparación con el placebo. Trastornos obsesivo-compulsivos. E clorhidrato de fluoxetina está indicado en el tratamiento del trastorno obsesivocompulsivo. La eficacia de la fluoxetina ha sido establecida en ensayos clínicos de 13 semanas de duración con pacientes ambulatorios con trastornos obsesivoscompulsivos cuyos diagnósticos correspondian estrechamente a la categoría de trastorno obsesivo-compulsivo del DSM-1II. 4.2. Posología y forma de administración: Depresión. Tratamiento inicial. La dosis inicial recomendada es de $20 \mathrm{mg}$ al día por la mañana. Después de varias semanas de tratamiento, y en caso de no observarse mejoría clínica, se puede considerar un aumento de la dosis. Las dosis por encima de $20 \mathrm{mg} /$ día deben administrarse dos veces al día (por ejemplo, por la mañana y al mediodia), y no debe excederse de la dosis máxima de $80 \mathrm{mg} / \mathrm{d}$ ía. Como sucede con otros antidepresivos, para que se alcance el efecto antidepresivo total, puede necesitarse un tiempo de 4 ó más semanas de tratamiento. Mantenimiento, continuación, tratamiento prolongado. No existen datos suficientes para poder hacer una recomendación en cuanto al tiempo que debe mantenerse el tratamiento de las personas tratadas con fluoxetina. En general, los episodios agudos de depresión necesitan varios meses de farmacoterapia sostenida. No se conoce si la dosis utilizada para inducir la remisión es idéntica a la que se necesita para mantener la eutimia. Bulimia nerviosa. La dosis recomendada es de 60 $\mathrm{mg}$ al día. Trastornos obsesivo-compulsivos. La dosis de $20 \mathrm{mg} / \mathrm{dia}$ a $60 \mathrm{mg} / \mathrm{día}$ es la dosis recomendada para el tratamiento del trastorno obsesivo-compulsivo. Los pacientes que recibieron 40 ó $60 \mathrm{mg}$ de fluoxetina en los ensayos clínicos de estudio de esta indicación, tendieron a mostrar un comienzo de la eficacia más temprana que los que recibieron $20 \mathrm{mg}$ de fluoxetina. Debido a que el trastorno obsesivo-compulsivo es una patología crónica, es razonable considerar el mantenimiento del tratamiento una vez que el paciente haya respondido al mismo. La eficacia de fluoxetina durante un tiempo mayor a trece semanas no ha sido sistemáticamente evaluada. Por lo tanto, el médico deberá reevaluar la utilidad a largo plazo de fluoxetina en cada paciente. En cualquiera de las indicaciones, la dosis de clorhidrato de fluoxetina no debería exceder de $80 \mathrm{mg}$ diarios. Uso en pediatría: no se recomienda el uso de fluoxetina en niños dado que su seguridad y efectividad no han sido establecidas. Uso en pacientes de edad avanzada: se recomienda una dosis diaria de $20 \mathrm{mg}$. Insuficiencia hepática: se deben utilizar dosis menores o menos frecuentes. Insuficiencia renal: en pacientes con insuficiencia renal grave, se recomienda la administración de dosis menores o menos frecuentes. Forma de administración: PROZAC se administra por via oral. 4.3. Contraindicaciones: Hipersensibilidad a fluoxetina. Fluoxetina no deberá administrarse en combinación con un inhibidor de la monoaminooxidasa (IMAO), ni tampoco durante los 14 días posteriores a la suspensión del tratamiento con un IMAO. Debido a que fluoxetina y su principal metabolito tienen vidas medias largas de eliminación, deberá pasar un mínimo de 5 semanas entre la suspensión de fluoxetina y el comienzo del tratamiento con IMAOs. 4.4. Advertencias y precauciones especiales de empleo: Advertencias:-Algunos pacientes con erupción cutánea relacionada con fluoxetina, han desarrollado reacciones sistémicas serias, posiblemente relacionadas con vasculitis. Aunque de forma rara, se ha comunicado éxitus en asociación con estos eventos. El clorhidrato de fluoxetina se debe interrumpir cuando se presente una erupción cutánea u otro fenómeno aparentemente alérgico para el que no se haya identificado otra etiología posible. PROZAC ${ }^{\circ} 20 \mathrm{mg}$ líquido: "Este medicamento contiene azúcar (sacarosa) en su composición, $3 \mathrm{~g}$ por cada $5 \mathrm{ml}$ aproximadamente, por lo que tendrán que tenerlo en cuenta las personas pacientes al comienzo del tratamiento ya que la posibilidad de un intento de suicidio es inherente a la depresión, y puede persistir hasta que se produzca una remisión significativa. Se ha producido activación de la maníahipomanía en una pequeña proporción de pacientes tratados con fluoxetina. 4.5. Interacción con otros medicamentos y otras formas de interacción: Se recomienda precaución si se requiere la utilización concomitante de clorhidrato de fluoxetina con otros medicamentos activos a nivel del sistema nervioso central, incluyendo el litio. Puede haber tanto aumento como disminución de los niveles de litio cuando se utiliza conjuntamente con fluoxetina. Se han comunicado casos de toxicidad por litio. Se deben vigilar los niveles de litio cuando ambos medicamentos se administren concomitantemente. Pacientes con dosis estables de fenitoina, han presentado aumento de las concentraciones plasmáticas de fenitoína y toxicidad clínica por fenitoina, tras iniciar tratamiento concomitante con fluoxetina. Se han observado incrementos superiores a dos veces las concentraciones plasmáticas de otros antidepresivos heterocíclicos, que previamente presentaban niveles plasmáticos estables, cuando se ha administrado fluoxetina asociada a estos agentes. El tratamiento concomitante con medicamentos que son metabolizados por el isoenzima P450IID6 (Ilecainida, encainida, vinblastina, carbamacepina y antidepresivos tricíclicos) puede requerir dosis más bajas de las habituales tanto de fluoxetina como del otro medicamento. En pacientes con diabetes, se ha producido hipoglucemia durante el tratamiento con fluoxetina e hiperglucemia tras la suspensión. Puede ser necesario el ajuste de la dosis de insulina y/o del agente hipoglucemiante oral. Ver también apartado: Contraindicaciones. 4.6. Embarazo y lactancia: Categoría B1: no se ha establecido la seguridad de este medicamento en mujeres embarazadas. Los estudios en animales de experimentación no indican efectos dañinos directos o indirectos respecto al desarrollo del embrión o feto, la gestación y el desarrollo peri y postnatal. Fluoxetina no debe ser utilizado en pacientes embarazadas, a no ser que sea claramente necesario. Fluoxetina es excretada en la leche materna, por lo que se deberá tener precaución cuando se administre clorhidrato de fluoxetina a mujeres durante la lactancia. 4.7. Efectos sobre la capacidad para conducir y utilizar maquinaria: Fluoxetina puede producir efectos adversos leves o moderados. Los pacientes deben tener precaución cuando manejen maquinarias peligrosas, incluyendo automóviles, hasta que exista una certeza razonable de que el tratamiento farmacológico no les afecta de forma adversa. 4.8. Reacciones adversas: Las reacciones más comúnmente observadas en asociación con el uso de fluoxetina y cuya frecuencia fue mayor del $2 \%$ y mayor que la de placebo, incluyen: ansiedad, nerviosismo, insomnio, somnolencia, astenia, temblor, sudoración, anorexia, náuseas, diarrea y mareo; reacciones menos frecuentes incluyen: cefalea, sequedad de boca, dispepsia y vómitos. También se han comunicado otras reacciones graves menos frecuentes (incidencia menor del $1 \%$ ) que incluyen: síncope, arritmia cardiaca, anomalías en las pruebas de función hepática, hipo e hipertiroidismo, aumento del tiempo de hemorragia, síndrome cerebral agudo y convulsiones. Ver también apartado: Advertencias y precauciones especiales de empleo. 4.9. Sobredosificación y su tratamiento: Signos y síntomas; las náuseas y vómitos son los síntomas predorninantes en la sobredosis de fluoxetina. Otros síntomas pueden ser: agitación, convulsiones, inquietud, hipomanía y otros signos de excitación del Sistema Nervioso Central. Desde su comercialización, las comunicaciones de casos de muerte atribuidas a una sobredosis de fluoxetina sola, han sido extremadamente raras. Hasta diciembre de 1987, se habian comunicado dos muertes entre 38 comunicaciones de sobredosis aguda con fluoxetina, tanto sola como en combinación con otros medicamentos $y / 0$ alcohol. Una de las muertes ocurrió en un paciente que tomó una sobredosis de $1.800 \mathrm{mg}$ de fluoxetina en combinación con una cantidad no determinada de maprotilina. Las concentraciones plasmáticas de fluoxetina y maprotilina fueron de $4,57 \mathrm{mg} /$ y $4,18 \mathrm{mg} /$, respectivamente. En el otro caso, que 作 a las concentraciones siguientes: $1,93 \mathrm{mg} / \mathrm{l}$ de fluoxetina, $1,10 \mathrm{mg} / \mathrm{l}$ de norfluoxetina, $1,80 \mathrm{mg} /$ de codeina y $3,80 \mathrm{mg} / \mathrm{l}$ de temazepam. Tratamiento: se recomienda tratamiento sintomático y de apoyo. El empleo de carbón activado con sorbitol, puede ser tan o más efectivo que la emesis o el lavado gástrico y debería ser considerado en el tratamiento de la sobredosis. Las convulsiones inducidas por fluoxetina que no se resuelvan de manera espontánea pueden responder a diazepam. En el manejo de la sobredosis se debe considerar la posibilidad de que el paciente haya ingerido una diversidad de fármacos. En pacientes que estén tomando fluoxetina o recientemente la hayan tomado e ingieran una cantidad excesiva de antidepresivos se debería proceder con especial cuidado, ya que en tales casos se podría aumentar la posibilidad de secuelas clínicamente significativas y aumentar el tiempo requerido de observación médica estrecha del paciente. 5. PROPIEDADES FARMACOLóGICAS: 5.1. Propiedades farmacodinámicas. Fluoxetina es un medicamento de acción antidepresiva cuyo mecanismo de acción parece estar relacionado con la inhibición de la recaptación de serotonina por las neuronas del sistema nervioso central. 5.2. Propiedades farmacocinéticas. Después de 6 a 8 horas de una dosis oral única se observan concentraciones pico plasmáticas de fluoxetina. Los alimentos no parece que afecten la biodisponibilidad sistémica de fluoxetina. Fluoxetina se distribuye ampliamente por el cuerpo uniéndose mucho a proteínas plasmáticas. La vida media de eliminación de fluoxetina es de 4-6 dias, siendo la de su metabolito activo de 4-16 días (ambas pueden aumentar en pacientes con deficiencia del sistema P450IID6). Esto asegura una presencia significativa de estos principios activos en el organismo durante el uso mantenido. Se alcanzan concentraciones plasmáticas en estado de equilibrio después de dosificar varias semanas. Fluoxetina es metabolizada fundamentalmente en el hígado a norfluoxetina y a otros metabolitos. La vía principal de eliminación es el metabolismo hepático dando lugar a metabolitos inactivos que son excretados por el riñón. La insuficiencia hepática puede afectar la eliminación de fluoxetina. Se puede producir acumulación adicional de fluoxetina o de sus metabolitos en pacientes con alteración severa de la función renal. 5.3. Datos preclínicos sobre seguridad. No existe evidencia de que el uso del clorhidrato de fluoxetina cause carcinogénesis, mutagénesis o alteraciones de la fertilidad. De seis perros que recibieron una sobredosis oral de fluoxetina, cinco experimentaron convulsiones generalizadas. Estas convulsiones cedieron en forma inmediata al administrar un bolo intravenoso de una dosis veterinaria estándar de diazepam. En este estudio de corto plazo, la concentración más baja de fluoxetina con la cual se presentaron convulsiones, fue tan solo el doble de la concentración plasmática máxima alcanzada en seres humanos que ingirieron una dosis de $80 \mathrm{mg} / \mathrm{día}$ crónicamente. En ratas, ratones y perros tratados con fluoxetina de forma crónica, se observo un aumento de los fosfolipidos en ciertos tejidos. Este efecto es reversible después de la interrupción del tratamiento. Se ha observado acumulación de fosfolípidos en animales tratados con diversos medicamentos anfifílicos catiónicos, incluyendo fenfluramina, imipramina y ranitidina. La importancia de este efecto en los seres humanos es desconocida. 6. DATOS FARMACÉUTICOS: 6.1. Lista de excipientes. PROZAC $20 \mathrm{mg}$, cápsulas: almidón de maíz y dimeticona. PROZAC ${ }^{\bullet} 20 \mathrm{mg}$, líquido: sacarosa, ácido benzoico, glicerol, saborizante de menta y agua purificada. PROZAC $20 \mathrm{mg}$, comprimidos: sacarina sódica, celulosa microcristalina, manitol, sorbitol, aroma de anís, aroma de peppermint, sílice coloidal, almidón de maiz, fumarato sódico de estearilo y polividona. PROZAC• $20 \mathrm{mg}$, sobres: sacarina sódica, aroma de anis, aroma de peppermint, manitol y sorbitol. 6.2. Incompatibilidades. No procede. 6.3. Periodo de validez. Cápsulas: tres años a temperatura ambiente. Solución: dos años a temperatura ambiente. Comprimidos: dos años a temperatura ambiente. Sobres: dos años a temperatura ambiente. 6.4. Precauciones especiales de conservación. No se requieren condiciones especiales para su conservación. 6.5. Naturaleza y contenido del recipiente. Presentaciones. PROZAC $20 \mathrm{mg}$ cápsulas: envase con 14 cápsulas para uso oral (982231); P.V.P. 2.384 Ptas., P.V.P. IVA 4- 2.479 Ptas., PROZAC $20 \mathrm{mg}$ cápsulas: envase con 28 cápsulas para uso oral (759811); P.V.P. 4.711 Ptas., P.V.P. IVA 4- 4.899 Ptas., PROZAC $20 \mathrm{mg}$ liquido: envase con $70 \mathrm{ml}$ para uso oral (692772); P.V.P. 2.384 Ptas., P.V.P. IVA 4- 2.480 Ptas., PROZAC $^{\circ} 20 \mathrm{mg}$ liquido: envase con $140 \mathrm{ml}$ para uso oral (651364); P.V.P. 4.363 Ptas., P.V.P. IVA 44.538 Ptas., PROZAC $20 \mathrm{mg}$ comprimidos: envase con 14 comprimidos para uso oral (982223); P.V.P. 2.384 Ptas., P.V.P. IVA 4- 2.479 Ptas., PROZAC 20 mg comprimidos: envase con 28 comprimidos para uso oral (651356); P.V.P. 4.711 Ptas., P.V.P. IVA 4- 4.899 Ptas., PROZAC $20 \mathrm{mg}$ sobres: envase con 14 sobres para uso oral (668921); PVP 2.384 Ptas., PVP IVA 4- 2.480 Ptas., PROZAC ${ }^{\circ} 20 \mathrm{mg}$ sobres: envase con 28 sobres para uso oral (668913); PVP 4.710 Ptas., PVP IVA 4- 4.899 Ptas., El envase de venta contiene un prospecto dirigido al paciente. 6.6. Instrucciones de uso/ manipulación. Comprimidos: se recomienda la administración de los comprimidos o bien disueltos en un poco de agua o ingeridos enteros. Sobres: disolver cada sobre en medio vaso de agua. 7. FECHA DE APROBA FICHA TÉCNICA: junio 1997. DISTA, S. A. Avda. de la Industria, 30. Zona Industrial. 28108 Alcobendas, Madrid. Con receta medica. 
¿S a b e dónde puede e n c o n t r a r c o m p ri midos 1 a experiencia de 20 a nos investig a d o e n D e presión, la se guridad d e má s de $30 \mathrm{millones}$ de tratamientos en los cinco continentes y la confianza continuada de miles de profesionales
y pacientes en
el mundo
entero?

AQUí.

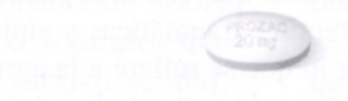

\section{COMPRIMIDOS.}
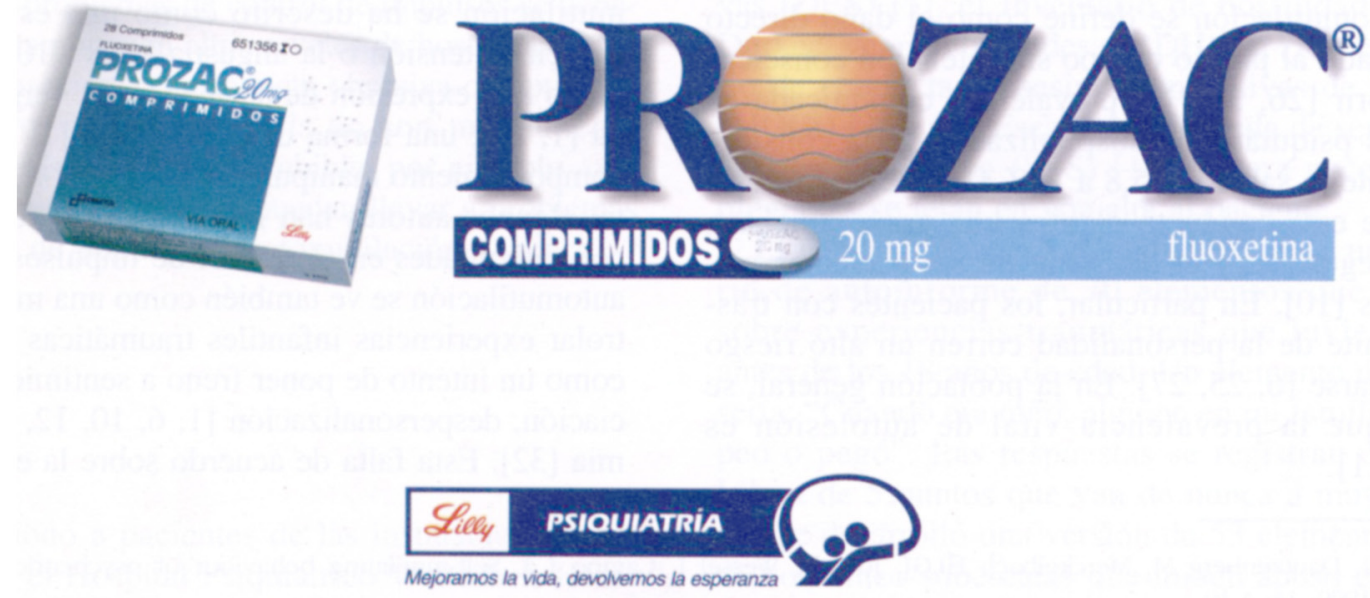

^ Prozac comprimidos ha sido investigado y desarrollado por Lilly España. 